Cultures \& Conflits

12 | hiver 1993

L'action collective : terrains d'analyse

\title{
Le communautarisme et la question de la reconnaissance
}

Ayse Ceyhan

\section{(2) OpenEdition}

1 Journals

\section{Édition électronique}

URL : http://journals.openedition.org/conflits/447

DOI : $10.4000 /$ conflits.447

ISSN : 1777-5345

Éditeur :

CCLS - Centre d'études sur les conflits lilberté et sécurité, L'Harmattan

Édition imprimée

Date de publication : 15 décembre 1993

ISSN : 1157-996X

Référence électronique

Ayse Ceyhan, «Le communautarisme et la question de la reconnaissance », Cultures \& Conflits [En ligne], 12 | hiver 1993, mis en ligne le 14 mars 2006, consulté le 30 mars 2021. URL : http://

journals.openedition.org/conflits/447 ; DOI : https://doi.org/10.4000/conflits.447

Ce document a été généré automatiquement le 30 mars 2021.

Creative Commons License 


\title{
Le communautarisme et la question de la reconnaissance
}

\author{
Ayse Ceyhan
}

Comment les sociétés démocratiques, dont le principe de base est le traitement égal de tous les citoyens, peuvent-elles reconnaître les identités différentes? Aujourd'hui des communautés ou des groupes se distinguant par des convictions philosophiques, religieuses ou morales, ainsi que par des spécificités culturelles, des modes de vie et des pratiques sexuelles, vivent sur le même territoire. Quels principes politiques vont régir leur coexistence tout en reconnaissant leurs identités différentes? John Rawls et Jurgen Habermas répondent à cette question fondamentale en proposant un système fondé sur un minimum commun qui unit les différences, les principes de la justice et les droits de l'homme pour l'un, la communication et le patriotisme constitutionnel pour l'autre. Mais, aux yeux des communautaristes, ces modèles fondés sur l'universalisme et la conception kantienne de l'autonomie du sujet sont incapables de reconnaître les diverses identités et produisent des impasses éthico-politiques difficiles à surmonter ${ }^{1}$. Développée aux Etats-Unis et au Canada depuis la fin des années soixante-dix, la pensée communautarienne est un courant qui tend à devenir presqu'une nouvelle idéologie outre-Atlantique. Il mobilise non seulement les philosophes, mais aussi les politologues et les sociologues dont Amitai Etzioni, considéré de nos jours comme "le "gourou" du mouvement communautarien. Contre les excès et les manifestations triviales de l'individualisme libéral, le communautarisme propose un retour vers les valeurs familiales, religieuses et culturelles ainsi que la reconstruction du lien social sur le modèle du lien intercommunautaire. Loin d'être limitée aux seuls cercles intellectuels, cette critique du modèle libéral a inspiré un certain nombre de mouvements de contestation aux Etats-Unis, comme le phénomène du "Political Correctness" (P.C.) sensible à la politique identitaire et dont l'expression la plus immédiate est d'ordre linguistique. Ainsi doit-on éliminer du langage quotidien tout ce qui a trait à la discrimination raciale ou sexiste: on ne dira plus "Blacks" mais "Afro-Americans", "Indians" mais "Native-Americans", "chairman" mais "chairperson", "immigrants" mais "minorities" etc. Il a par ailleurs inspiré le mouvement "Affirmative Action" qui se propose de promouvoir la solidarité communautaire en mobilisant les membres d'un 
groupe autour des injustices spécifiques qu'ils subissent et en les incitant à retrouver leur culture propre étouffée par la culture dominante. Au Québec, il soutient le mouvement de la défense de la langue et de la culture françaises et a pris une tournure politique affirmant des revendications séparatistes. Aujourd'hui face à la crise de l'Etatprovidence, et à l'incapacité des politiques sociales à résoudre les problèmes et les conflits, le communautarisme apparait aux yeux de ses tenants comme une alternative pour ce qui concerne par exemple les problèmes d'immigration-intégration, d'éducation, de santé, de la ville, de la représentation des groupes minoritaires tels que les homosexuels, etc. Dans quel sens faut-il voir cette prétention? Dans le sens d'une dynamique de la fragmentation s'opposant à la dynamique de globalisation ou d'intégration? Dans celui d'un mouvement totalisant, expression d'un holisme particulariste ? Ou bien s'agit-il d'un phénomène plus complexe mettant en interaction plusieurs dynamiques qui sont parfois prises dans un paradoxe difficile à saisir?

\section{CRITIQUE DE L'UNIVERSALISME ET DE SON MODELE DE LA RECONNAISSANCE}

Les sociétés libérales et démocratiques ont pour principe de base la représentation égale de tous les citoyens sans distinction de race, de culture, de sexe et d'origine sociale. Mais pour Charles Taylor, un des principaux théoriciens du communautarisme, et un de ceux qui a le plus écrit sur le sujet, ce principe universel hérité des Lumières a aujourd'hui atteint ses limites et ne peut plus répondre "à la demande et au besoin de la reconnaissance de différentes identités culturelles". Il engendre la non-reconnaissance ou la méconnaissance de ceux qui ne partagent pas les mêmes valeurs que la majorité et devient ainsi la cause de nombreux conflits sociaux ${ }^{2}$. Aussi c'est au nom de l'universalisme et de son principe de neutralité de la sphère publique, que les identités seraient méconnues dans les sociétés libérales atomisées ${ }^{3}$. C'est en dénonçant le modèle rousseauiste du contrat social que Taylor construit sa critique du modèle libéral. Affirmant que "le libéralisme rousseauiste de droits égaux ne donne lieu qu'à une reconnaissance très limitée des droits culturels spécifiques"4 , il lui reproche d'exclure toute politique de différenciation et d'imposer une politique d'homogénéisation au nom de la volonté générale. Dans "Multiculturalism and The Politics of Recognition"5 , il introduit la problématique de la reconnaissance en faisant référence au développement des notions d'honneur et de dignité. Il explique que nous sommes passés d'une société où le concept principal, à partir duquel les êtres humains se faisaient reconnaître, était l'honneur à une société où le concept principal est celui de la dignité. Si l'honneur traditionnel était un concept hiérarchique impliquant des privilèges, la dignité telle qu'on la conçoit dans la société moderne est un principe égalitaire et un concept universel. Les conséquences de cette promotion de l'idéal égalitaire concernent précisément la dimension de l'identité, non plus au sens de l'identité qui lie le groupe, mais au sens de ce qui définit chaque individu, au sens de ce qui donne valeur et sens à sa vie. L'identité des individus, l'identité qu'ils se donnent dans une ère d'égalité doit être une identité "engendrée intérieurement" (c'est à dire liée à une structure déjà préexistante) ${ }^{6}$. Ce n'est donc pas une identité qui leur est attribuée d'après leur position et leur situation dans un ordre hiérarchique. Or, affirme Taylor, dans le modèle rousseauiste du contrat social l'identité n'est pas reconnue comme étant "engendrée de l'intérieur", mais attribuée de l'extérieur. Aussi, la demande de reconnaissance ne 
pourrait-elle être satisfaite qu'après qu'elle eut été socialement et politiquement disciplinée ${ }^{7}$. Ceci instaure à ses yeux un système totalisant, qui méconnaît toute différence et spécificité identitaire ${ }^{8}$. Face au paradigme contractualiste Taylor propose un modèle de la reconnaissance qui peut se résumer à deux aspects : le premier réside dans la nécessité d'un point ou d'une base d'unité où les gens pourront se sentir unis dans un même projet (la communauté). Le second réside dans la négociation des identités par reconnaissance avec les autres, reconnaissance signifiant "acceptation de valeur égale". Il est important de noter ici que Taylor appelle à une "conversation" entre diverses identités : "cette identité devrait se forger en conversation avec d'autres et implique une certaine reconnaissance" 9 . Ce qui le distingue nettement des autres communautaristes plus radicaux qui comme nous le verrons plus loin passent sous silence cet aspect fondamental de la question. Afin de ne pas tomber dans les pièges du cosmopolitisme libéral, Taylor propose de poser d'abord la reconnaissance de "la survivance culturelle" comme objectif légitime sur le plan juridique de sorte que les différentes cultures puissent se défendre dans des limites raisonnables "sans compromettre les principes politiques fondamentaux"10. Il faudra ensuite passer à "la reconnaissance de la valeur égale de chaque culture". Là, il ne s'agit pas de s'intéresser à une culture autre que la sienne juste par curiosité ou volonté intellectuelle, mais de faire un "travail d'élimination d'images défavorisantes ou négatives imposées à celle-ci par la culture occidentale dominante afin de parvenir à un "jugement de valeur égale"11. Ce qui se traduit sur le plan de l'enseignement, par exemple, par la réécriture des livres scolaires selon le critère multiculturel, éliminant les références dévalorisantes. Pour les communautaristes, la raison ultime de la méconnaissance se trouve au fond dans l'idée de l'autonomie du sujet qui est à la base de la conception libérale de la société. Faisant valoir le caractère faux et trompeur de l'idéal kantien d'autonomie, ils affirment que l'individu abstrait n'existe pas ${ }^{12}$, le sujet politique ne peut être appréhendé en faisant abstraction de ses déterminations sociales et surtout culturelles qui nourrissent son individualité et en particulier sa conception de la "vie bonne". Car pour eux les sujets se trouvent déjà "enchâssés" dans un horizon de valeurs historico-culturelles à partir duquel ils se comprennent eux-mêmes ${ }^{13}$. Ce qui peut se résumer ainsi : "mon bien en tant qu'homme ou femme est le seul et le même bien que celui des autres avec qui je suis lié dans la communauté". Dans "Liberalism and the Limits of Justice" Michael Sandel affirme contre Rawls que nous ne pouvons pas être des agents entièrement autonomes, indépendants dans le sens où notre identité n'est jamais liée à nos projets et attaches ${ }^{14}$. De même, critiquant la liberté trop "libérale" de John Rawls, Joseph Raz proche des communautaristes, pense qu'être libre c'est aussi être attaché à des valeurs, à une tradition et non pas seulement être neutre ou refuser toute hiérarchie de valeurs morales et sociales ${ }^{15}$. Ainsi, pour les communautaristes, la liberté doit-elle avoir un caractère communautaire se manifestant dans la manière dont l'individu participe et contribue aux pratiques communautaires de la sociétée ${ }^{16}$.

\section{LA PERSPECTIVE COMMUNAUTARIENNE}

La pensée communautarienne puise ses sources dans la tradition aristotélicienne qui avait été abandonnée par les théories modernes individualistes et les théories du contrat social, ainsi que dans des auteurs médiévaux tels que John de Salisbury et Marsile de Padoue qui se sont inspirés d'Aristote. Contre la pensée libérale qui affirme que la société peut accepter une diversité des conceptions du bien, les 
communautaristes proposent la politique du bien commun (Taylor, Sandel, MacIntyre) qui repose sur les modèles traditionnels de la "vertu civique" (MacIntyre). On peut dégager deux caractères fondamentaux du modèle communautarien: son caractère narratif et son caractère fonctionnel.

Le communautarisme narratif Dans "After Virtue. A Study of Moral Theory" MacIntyre explique que la société doit être définie en terme de bien substantiel ou d'ensemble de biens qui génère une identité civique dans laquelle tous les membres sont enchâssés ${ }^{17}$. Il voit là un lien entre orientation au bien et la notion d'identité narrative qu'il considère comme le trait constitutif de l'identité personnelle. Ce qui veut dire que les sujets ne peuvent faire autrement que de s'orienter par rapport au bien et ainsi déterminer la direction de leurs vies et que de ce fait, ils doivent comprendre leurs vies dans la forme narrative cimentée par une histoire et une tradition communes ${ }^{18}$. On peut dire ici avec Jean-Marc Ferry que le communautarisme fait le choix d'une "identité narrative qui organise sa compréhension du monde sur des catégories de l'Evénement et du Destin qui sont celles du récit épique et du mythe"19. Aussi, lui est-il difficile de souscrire aux conditions "d'un espace public moderne structuré par l'argumentation sans renoncer à l'élément substantiel de l'identité structurée en dominante par les vertus de la narration" ${ }^{20}$. Il faudrait également noter que, si pour les communautaristes, avec la forme narrative, l'identité personnelle des individus est la même pour tous, leur identité publique n'est pourtant pas exactement identique. Dans le modèle communautarien que Habermas appelle "modèle totalisant d'une collectivité dans laquelle le citoyen serait tout entier incorporé" ${ }^{21}$, l'identité publique des membres de la société n'est pas formée en référence à un modèle de vertu civique identique pour tout le monde, mais forgée par le groupe et la communauté auxquels ils appartiennent et par le rôle ou la position qu'ils occupent dans la société. Nous touchons ici à un aspect important du communautarisme, à savoir son caractère fonctionnel.

Le communautarisme fonctionnel De quoi est composée la communauté ? Dans un article publié dans l'American Political Science Review, Cary J. Nederman remonte au Moyen-Age latin pour retrouver les sources de ce qu'elle appelle le "fonctionnalisme communal" duquel dériverait le modèle communautariste de la société22. Selon cette approche, la communauté n'est composée ni d'individus ni de citoyens, mais de groupes fonctionnels dont la position est déterminée selon la nature de leurs contributions à l'ensemble communautaire ${ }^{23}$. Le communautarisme fonctionnel se caractérise par deux aspects : un aspect organique et un aspect civique. Pour son aspect organique, il puise ses sources chez John de Salisbury (12ème siècle) philosophe et religieux, auteur de "Policratus", pour qui l'appartenance à la communauté est la conséquence de la contribution d'un groupe à travers la performance d'une fonction destinée au bien-être de la communauté laquelle, est conçue suivant le modèle du corps humain. Pour son aspect civique c'est chez Marsile de Padoue (14ème siècle) philosophe et polémiste anticlérical, auteur du "Défenseur de la paix" qu'il trouve son inspiration. En se référant à l'Ethique d'Aristote, Marsile de Padoue énumère six groupes fondamentaux qui constituent une communauté parfaite: agriculteurs, artisans, militaires, commerçants, religieux, juges. Cependant ces groupes n'exercent pas leurs fonctions isolément, mais en relation les uns avec les autres et par rapport au bien commun. Ce qui génère une identité civique. On peut dire que le fonctionnalisme communal pourrait être une conception purement organique s'il n'y avait pas cette référence à Marsile de Padoue, qui permet d'intégrer les concepts de vertu civique et 
d'identité dans le modèle de la communauté. De nos jours, le communautarisme fonctionnel est de plus en plus présenté comme un modèle de représentation de ce qu'on appelle globalement les minorités : immigrés, homosexuels, groupes alternatifs etc. Faut-il favoriser la représentation des immigrés et des homosexuels sous forme de communautés ou de groupes? Doit-on favoriser le développement d'une identité communautaire homosexuelle qui s'inscrive comme telle sur le plan politique? Les "gays" américains ont depuis longtemps opté pour cette option et mettent aujourd'hui au défi l'administration Clinton pour qu'elle les reconnaisse comme groupe distinct (par exemple dans l'armée) ayant des droits spécifiques.

En ce qui concerne la représentation des immigrés, à l'opposé du modèle intégrationiste français, le Royaume-Uni, s'inspirant du modèle de la mosaïque qui se développe aux Etats-Unis, a élaboré le modèle de l'insertion communautaire. Partant de l'idée que l'intégration individuelle est impossible et qu'il n'y a pas d'universalité citoyenne, celui-ci propose de reconnaître les immigrés en fonction de leur appartenance ethnique ou religieuse en tant que communautés différentes. Au fond, comme l'explique Gilles Kepel, le Royaume-Uni veut édifier une société pluri-ethnique, multiculturelle, caractérisée à terme par la différence des droits et renonçant à toute universalité de valeurs ${ }^{24}$. La question devient plus pertinente pour les communautés qui veulent privilégier le rôle de la religion, telles les communautés musulmanes. $\mathrm{Ne}$ risque-t-on pas de favoriser par là le développement d'un islamisme radical qui s'exprime sur des bases communautaires refusant toute idée de laïcité et de droit universel ? Est-il possible de faire coexister le droit musulman et le droit universel laïc quand le premier refuse la validité du second? Par ailleurs sur le plan social, voulant faire reconnaître les droits des minorités, ce modèle ne risque-t-il pas en fait de pousser les étrangers à s'enfermer dans des communautés distinctes souvent défavorisées? Ces questions nous conduisent à deux réflexions : premièrement, au fond, le communautarisme fonctionnel demeure trop restrictif dans sa conception de la diversité de biens humains. Nous rappellerons avec Cary Nederman que John de Salisbury et Marsile de Padoue adoptent une définition purement publique de la fonction: ce qui compte comme fonction c'est ce qui contribue au bien-être de la communauté25. Ce qui exclut les fonctions privées (la famille) de la sphère du bien commun et plus troublant encore élimine de la vie publique toutes les personnes ou groupes qui n'ont pas de fonction: les chômeurs et les SDF (sans domicile fixe). Deuxièmement, en s'appuyant au départ sur les thèses identitaires/différentialistes, le communautarisme ne tombe-t-il pas dans un paradoxe : le droit à la reconnaissance égale sort d'une expression de la différence. Ce qui risque de le réduire à un culturalisme différentiel où la validité des jugements moraux ne dépendrait plus que de ressources culturelles valables à une époque donnée, dans un lieu donné.

\section{LES AMBIGUITES DU MODELE DE LA RECONNAISSANCE}

L'examen des textes communautariens nous fait penser que même si les communautariens posent la problématique de la reconnaissance, elle n'est pour eux qu'un objectif secondaire, l'essentiel de leur analyse restant centrée sur la représentation de la communauté comme macrosujet et sur l'élaboration d'une approche normative de l'identité communautaire. On se demande alors quelle est leur 
attitude face à la société moderne où coexistent plusieurs identités, plusieurs biens, plusieurs conceptions de "vie bonne", enfin plusieurs interactions multipolaires. Amitai Etzioni remarque à juste titre que le communautarisme en raison de sa critique virulente du libéralisme et du statut précaire qu'il accorde à l'individu peut nous apparaître comme une philosophie collectiviste ${ }^{26}$. Il ne sépare pas clairement le soi de son rôle social et fait dépendre la validité des jugements moraux des ressources culturelles valables à une époque et à un contexte donnés. Ainsi, dans la perspective de MacIntyre par exemple, la communauté est restreinte, éthniquement homogène et intégrée par une tradition. En raison de l'impossibilité de transcender les rôles sociaux, toute idée de critique sociale est quasiment absente et le pluralisme est considéré comme une "menace". Cependant, il serait erroné d'affirmer que tous les communautariens sont aussi radicaux que MacIntyre. On pourrait évoquer par exemple Walzer, qui propose la notion "d'égalité complexe". Dans Spheres of Justice, il affirme qu'il n'y a pas un bien commun unique mais plusieurs biens communs ayant chacun une signification sociale différente ${ }^{27}$. Puisque ces biens sont multiples, ils ont différents principes de distribution, aussi, doivent-ils être gardés autonomes. D'où l'idéal de communauté décomposée en une pluralité de sous-communautés. Cependant, l'individu a-t-il le droit de choisir librement parmi les biens autonomes et peut-il passer d'une sous-communauté à une autre ? C'est là une véritable question et Walzer ne semble pas y répondre. Chez Taylor qui centre sa problématique sur la reconnaissance du Québec, le rapport au pluralisme est mieux posé. Contre le modèle libéral qui ne se réclame, selon le principe de la neutralité d'aucune culture particulière et protège les droits et le bien-être de tout le monde, il propose un modèle de gouvernement qui "nourrit" une culture particulière tout en protégeant les droits et le bien-être de tout le monde, y compris de ceux qui n'adhèrent pas à celle-ci. Car pour lui, cette prétendue neutralité ne représente au fond que les préoccupations de l'homme blanc et hétérosexuel. D'ailleurs affirme-t-il "le libéralisme occidental ne peut pas et ne doit pas prétendre à une totale neutralité, puisque la séparation de l'Etat et de l'Eglise puise ses sources dans les premiers temps de la civilisation chrétienne" ${ }^{28}$. Dans le modèle taylorien il y aurait ainsi d'un coté la protection des libertés fondamentales pour tout le monde, de l'autre, la préservation d'une conception particulière de la vie bonne sans que celle-ci soit considérée comme nuisible à ceux qui ne la partagent pas $^{29}$. La question qui se pose alors est de savoir si la sauvegarde d'une culture particulière peut devenir condition d'accès à l'universel. Prenons le cas du Québec pour voir de près les ambiguïtés d'une telle proposition. Quand Taylor parle d'un modèle de gouvernement qui "nourrit" une culture particulière, il pense naturellement à la revendication québécoise de la "survie culturelle" (survie de la langue et de la culture françaises). Pour les Québécois cette revendication ne doit pas rester marginale, mais doit être reconnue comme un "objectif légitime" de gouvernement ${ }^{30}$. Ainsi aurions-nous un gouvernement (Québec) qui nourrit une culture particulière (la culture française) tout en respectant et reconnaissant les droits et le bien-être de tout le monde (anglophones, indiens Mohawks, immigrés). Pour Taylor, dans cet exemple, il y a un bien collectif : la préservation de l'identité francophone qui peut toutefois être atteinte sans sacrifier les autres, car le bien n'exclut pas le juste, c'est à dire le respect égal des minorités. Ainsi dans son raisonnement il ne s'agit pas d'obliger tout le monde à parler français, mais il n'empêche que ce bien collectif est le français et la fameuse Loi 101 le protège jalousement en obligeant les familles francophones et immigrées à envoyer leurs enfants à l'école francophone, en instaurant des quotas de langue pour la diffusion 
télévisuelle et radiophonique, en imposant le français dans les entreprises de plus de cinquante personnes etc. Parallèlement à la question linguistique, il faudra également considérer le poids de l'histoire qui accentue le désir de la revanche, de se faire connaître comme "société distincte" et d'imposer ses propres règles. Et, tout ceci a pour conséquence le départ massif des anglophones malgré la protection que leur procure le Charter of Rights (la Charte des droits). Face à ces restrictions et interdits on se demande alors si le modèle communautarien présenté comme "libérateur" ne devient-il pas lui-même exclusionniste, produisant des tensions et un renforcement des formes de violence symbolique et institutionnalisée ? On peut dire que la question de la validité du modèle communautarien reste ouverte. Si le modèle de l'universalisme que les communautariens critiquent, par sa non-reconnaissance des différences entraîne des formes de discrimination et de violence, le modèle communautarien, favorisant le particularisme, et plus généralement les constructions identitaires porteuses de sens ne comporte-t-il pas lui aussi des risques de division, de violence et d'affrontement intercommunautaire? Observant le communautarisme radical, déjà sur le plan de la construction du modèle, on peut repérer des signes annonciateurs de la violence symbolique et physique. En effet, l'idée de la reconnaissance de la valeur égale est souvent accompagnée par la référence aux Damnés de la Terre de Franz Fanon qui est devenu l'un des auteurs fétiches de la lutte multiculturelle. Dans ce livre, affirmant que l'arme majeure des colonisateurs était l'imposition par la force de leurs images sur les colonisés, Fanon recommande le recours à la violence pour se libérer des images dévalorisantes d'identité ainsi imposées. C'est ainsi que par exemple certaines branches du féminisme américain n'hésitent pas à utiliser la violence pour "arracher les femmes aux stéréotypes infamants produits par l'idéologie dominante" et leur permettre de trouver leur place dans la société et au sein de la famille. Le problème aujourd'hui pour une société comme les Etats-Unis est d'éviter la ghettoïsation et l'affrontement intercommunautaire tout en luttant contre l'individualisme excessif qui a engendré le "me generation" (génération moi). Dans son livre "A Different Mirror", Ronald Takaki montre combien l'animosité intercommunautaire a marqué l'histoire multiculturelle américaine, et comment par exemple les immigrants irlandais ont exploité les Noirs, comment les Hawaïens propriétaires de plantations ont attisé les tensions entre ouvriers philippins et japonais, comment de nos jours les tensions s'aggravent de plus en plus entre les communautés cubaines, mexicaines, coréennes et noires, etc. ${ }^{31}$ Les émeutes de Los Angeles de 1992 ont d'ailleurs montré que même en matière de griefs contre le gouvernement, les communautés n'hésitaient pas à s'en prendre à ellesmêmes. Ainsi, même s'ils se sentaient discriminés en matière d'attribution de subventions, plutôt que de se révolter contre le gouvernement, les Noirs ont préféré attaquer les Coréens qu'ils considéraient avoir été favorisés par les autorités gouvernementales. Sur le plan sociologique cet exemple montre que la dénonciation communautarienne de l'injustice sociale ou politique s'affirme par la sortie du jeu institutionnalisé en opposition avec la revendication universaliste qui est, elle, canalisée et institutionnalisée. Face à ce danger de dualisation et d'affrontement, la question essentielle reste de savoir comment instaurer le dialogue intercommunautaire sans tomber dans des conflits d'intérêt. Dans son raisonnement, Taylor affirme que les diverses identités ne peuvent avoir de projet commun, ou ne peuvent coexister au sein d'un Etat-nation si elles ne se reconnaissent pas mutuellement. Mais selon sa théorie, pour qu'une identité puisse exister, elle doit faire partie d'un projet commun communautaire, se sentir vraiment partie prenante d'un projet. Cependant, dans le 
cadre du modèle de la reconnaissance, comment assurer que le projet commun communautaire soit compatible avec le projet de cette identité ? Comment faire pour qu'il n'y ait pas de conflits? Nous avions évoqué au début l'accent que Taylor mettait sur la "conversation entre identités". Qu'entraîne cette conversation? En fait, sans le vouloir, Taylor ne nous pousse-t-il pas par là à nous référer au paradigme communicationnel et à la recherche d'un minimum commun fédérateur? On peut dire que les thèses de Taylor ne semblent pas être totalement antithétiques avec les thèses universalistes communicationnelles comme le sont par exemple celles de MacIntyre ou de Sandel. Face à un individualisme excessif et à un communautarisme radical, l'alternative réside peut-être dans une position médiane développée par Etzioni qui prend ses distances à l'égard d'un holisme particulariste et ethnocentrique ${ }^{32}$. Remarquant l'impossibilité du communautarisme radical à empêcher la formation d'un modèle monolithique fonctionnant comme un principe d'exclusion et d'intolérance, il propose avec son "I \& We paradigm" un modèle où serait possible une forme d'identité communautarienne qui postule la solidarité collective mais qui ne supprime pas pour autant la médiation de l'individu. Reste à savoir comment cet idéal pourrait être réalisé sur le plan social, surtout aux Etats-Unis où le communautarisme radical gagne de plus en plus du terrain.

\section{BIBLIOGRAPHIE}

Badie Bertrand, "Communauté, individualisme et culture" in P. Birnbaum et J. Leca (dir.), Sur l'individualisme, Presses de la FNSP, coll.Références, Paris, 1986. Black Anthony, Guilds and Society in European Political Thought from the Twelfth Century to the Present, Ithaca, Cornell University Press, 1984. Bouretz Pierre (dir.), La force du droit, Paris, éd. Esprit, 1991. Bruckner Pascal, "Faut-il être cosmopolite ?", Esprit, décembre 1992. Burtt Shelley, "The Politics of Virtue Today: A Critique and A Proposal", American Political Science Review, vol.87, n², 1993. Cladis Mark, Communitarian Defense of Liberalism: Emile Durkheim and Contemporary Social Theory, Stanford, Stanford University Press, 1993. Dewandre Nicole et Lenoble Jacques (dir.), L'Europe au soir du siècle, Paris, Editions Esprit 1992. Dumont Louis, Essai sur l'Individualisme, Paris Le Seuil, 1983. L'idéologie allemande. France-Allemagne et retour, Paris Gallimard 1991. Etzioni Amitai, The Moral Dimension, New York, Free Press, 1988. A Responsive Society, San Francisco, Jossey Bass Publishers, 1991. "Liberals and Communitarians" Partizan Rewiev, 1992. The Spirit of Community, First éd. New York, Crown Publishers, 1993. Ferry Jean-Marc, Les puissances de l'expérience, Paris, le Cerf 1991, 2 tomes. "Une idée moderne de la communauté. Linéaments d'un communautarisme méthodologique", Revue de l'Institut de Sociologie de l'Université Libre de Bruxelles, 1992 1-4, pp. 19-38. Habermas Jürgen, De l'éthique de la discussion, trad. par M. Hunyadi, Paris, Le Cerf 1992. Hassner Pierre, "Vers un universalisme pluriel ?", Esprit, décembre 1992. Javeau Claude, "Du mythe du contrat au mythe de la communauté", Revue de l'Institut de Sociologie de l'Université Libre de Bruxelles, 1992 1-4, pp.39-48. Lasch Christopher, "The Communitarian Critique of Liberalism" in Community in America, éd. Charles H. Reynolds and Ralph V. Norman, Berkeley, University of California Press 1988. Lure and Loathing: Essays on Race, Identity and the Ambivalence of Assimilation, éd. by Gerald Early, Penguin/Allen Lane, 1993. Macintyre Alasdair, After Virtue. A Study of Moral Theory, Notre Dame, Indiana University of Notre Dame Press 1981. Against Virtue, London, Duckworth, 1985 (2e éd). Whose Justice, Whose Rationality, London 1988, trad. fr. Quelle justice, quelle rationalité ?, 1992. First Principles, Final Ends and Contemporary Philosophical Issues, Minneapolis, 
Marquette University Press, 1990. Massey Douglas S., and Nancy A Denton, American Apartheid: Segregation and Making of the Underclass, Harvard University Press, 1993. Nederman Cary, "Freedom, Community and Function: Communitarian Lessons of Medieval Political Theory", American Political Science Review, vol. 86, n 4, décember 1992. Nispet Robert, The Quest for Community. A Study of the Ethics of Order and Freedom, (1re éd. Oxford University Press), Institute for Contemporary Studies, San Francisco, 1990. Raz Joseph, The Morality of Freedom, Oxford, Clarendon Press 1986. Ricoeur Paul, Soi-même comme un autre, Paris, Le Seuil, 1990. Sandel Michael, Liberalism and the Limits of Justice, Cambridge, Cambridge University Press, 1982. Schlesinger Arthur M.jr., The Desuniting of America. Reflexions on A Multicultural Society, W.W.Morton \& Company, New York-London, 1992 ; trad. fr. La désunion de l'Amérique, Paris, Liana Lévy, 1993. Takaki Ronald, A Different Mirror : A History of Multicultural America, Little Brown 1993. Taylor Charles, Sources of the Self, Cambridge, Mass., Harvard University Press, 1989. "Atomism", Philosophy and the Human Science. Philosophical Papers, t. 2, Cambridge University Press, 1985. "Alternative Features : Legitimacy, Identity and Alienation" in Late Twentieth Century Canada, éd. Alan Cairns and Cynthia Williams, Toronto, University of Toronto Press, 1986. Multiculturalism and the "Politics of Recognition", Princeton, Princeton University Press, 1992. The Ethics of Authenticity, Cambridge, Mass., Harvard University Press, 1992. "The Politics of Political Correctness", Partizan Review, Fall 1993. Tonnies Fernand, Gemeinschaft, Gesellschaft, Stuttgart, 1887, trad. fr., Communauté et Société, Paris PUF, 1954. Walzer Michael, The Spheres of Justice, London, Oxford University Press, 1983. Interpretation and Social Criticism, Cambridge, Mass., Harvard University Press, 1987. "Les deux universalismes", Esprit, décembre 1992. Wellmer Albrecht, "Modèles de la liberté dans le monde moderne", Critique, $n^{\circ}$ 505-506, juin-juillet 1989, pp. 506-539.

\section{NOTES}

1. Alasdair MacIntyre, After Vitue. A Study in Moral Theory, Londres, Duckworth, 2ème éd., 1987, pp. 220-222 et 244-257.

2. Ch. Taylor, Multiculturalism and "Politics of Recognition, Princeton, New Jersey, Princeton University Press, 1992, pp. 25-26.

3. Ch. Taylor, "Atomism" Philosophy and the Human Science, Philosophical Papers, Cambridge University Press, 1985, t. 2, p.187 et sq.

4. Multiculturalism..., op. cit., chap IV. p. 51.

5. op. cit.

6. Ch. Taylor, op. cit., pp. 26-35 ; "Quel principe d'identité collective ?" in Lenoble (J.) et Dewandre (N.) (dir.), L'Europe au soir du siècle, éd. Esprit, 1992, pp. 63-65.

7. Multiculturalism..., op. cit., p. 51.

8. Taylor reprend cette critique du contrat social dans The Ethics of Authenticity, Cambridge, Mass., Harvard University Press, 1992, p. 28.

9. Ch. Taylor, "Quel principe d'identité collective ?", op. cit., p. 64. 
10. Ch. Taylor, Multiculturalism..., op. cit., pp. 63-66.

11. Ch. Taylor, idem, p. 64.

12. A. Macintyre, op. cit., p. 321.

13. Ch. Taylor, The Sources of the Self, Cambridge, Mass., Harvard University Press, 1989 , p. 35.

14. Michael Sandel, Liberalism and the Limits of Justice, Cambridge University Press, 1982.

15. Joseph Raz, The Morality of Freedom, Oxford, Clarendon Press, 1986.

16. Ch. Taylor, "Atomism", op. cit., p. 187.

17. A.MacIntyre, op. cit.

18. Ch. Taylor, Sources of the Self, op. cit., p. 51.

19. J.- M. Ferry, Les puissances de l'expérience, Paris, Le Cerf, 1991, t. 1, p. 17 et pp. 103-110.

20. J.- M. Ferry, "pertinence du postnational", Esprit, novembre 1991, p. 92.

21. J. Habermas, "Citoyenneté et identité nationale. Réflexions sur l'avenir de l'Europe", in L'Europe au soir du siècle, op. cit. p. 27.

22. C. J. Nederman, "Freedom, Community and Function : Communautarian Lessons of Medieval Political Theory", American Political Science Review, vol. 86, n 43, 1992, pp. 19-35.

23. op. cit. p. 19.

24. G. Kepel, "L'islamisme militant tend à devenir le nouvel « empire du mal »", Le Monde, 16 février 1993.

25. C. Nederman, op. cit.

26. A. Etzioni, "Liberals and Communitarians", Partizan Review, 1992, pp. 220-221.

27. M. Walzer, Spheres of Justice, Oxford, 1983.

28. Ch. Taylor, Multiculturalism... op. cit.,p. 62.

29. idem, p. 59.

30. idem, p. 64.

31. R. Takaki, A Different Mirror : A History of Multicultural America, Little Brown, 1993.

32. A. Etzioni, op. cit.

\section{RÉSUMÉS}

Contre les excès de l'individualisme libéral et l'incapacité des politiques sociales de résoudre les problèmes, les communautariens proposent de reconstruire le modèle social sur le lien intercommunautaire. Critiquant le modèle libéral/universaliste de dénier toute diversité au profit de l'individu prétendument neutre mais qui ne représente en fait que les préoccupations d'un homme blanc et hétérosexuel, ils proposent un modèle de la reconnaissance centré sur la "reconnaissance de la valeur égale" de chaque culture. Mais face à la question multiculturelle, leur attitude n'est pas dépourvue ambiguïtés, ce qui entraîne des risques de discrimination, d'affrontement et de violence. 
INDEX

Mots-clés : démocratie, identité, Théorie politique, genre, Etat-nation 